\title{
Impact of Imperfect Channel Estimation Error and Jamming on the Performance of Decode-and-Forward Relaying
}

\author{
Khaled Eshteiwi, Georges Kaddoum, and François Gagnon \\ Department of Electrical Engineering \\ University of Quebec, ÉTS, LaCIME Laboratory \\ Montreal, Canada \\ Email: khaled-mohamed.eshteiwi.1@ens.etsmtl.ca, georges.kaddoum/francois.gagnon@etsmtl.ca
}

\begin{abstract}
In this paper, we analyze the bit error rate (BER) and the throughput of decode-and-forward (DF) relaying with imperfect channel estimation and in the presence of a jammer in the network. We consider a single DF relay scenario with orthogonal cooperative protocols and an active jammer during all the transmission phases attacking the relay and destination nodes in the network. In our analysis, we assume that the complex fading channel coefficients are estimated at the relay and destination levels with errors. We derive the error performance and the throughput of the network then we quantify the impact of channel estimation and the jamming power through the derivation of analytical performance expressions. Our simulation results confirm the accuracy of our analytical BER expression and quantify the performance degradation due to channel estimation and jamming signals. Also, simulation results show the performance of the throughput of the proposed system.

Index Terms-Cooperative Communications, Decode-andForward, Bit error rate, Imperfect Channel, Jamming.
\end{abstract}

\section{INTRODUCTION}

Cooperative wireless relaying and cooperative diversity networks have attracted the attention of the researchers for many years because of their efficient performance and achievement of maximum possible diversity networks. Also, many studies have been suggested to improve diversity and bandwidth utilization over fading channels. Cooperative wireless systems over Rayleigh fading channels with full or partial channel state information assumptions at either one of the nodes have been widely studied [1]-[3]. A simple cooperative model in which the direct link is unavailable with no PCSI (perfect channel state information) at the transmitter has been presented in [4]. In [4], the authors investigate the power allocation of the jammer when the jammer sends signals to both the relay and destination.

Altman et al. have studied in [5] the effect of the correlation between sub-carrier channel fading gains and the available information on the sender side when a jammer is present in the network. Furthermore, they have applied a game theory approach such as zero-sum game rule between nodes and the jammer to measure the signal-to-interference-plus-noise ratio (SINR). In [6], some of the available power at both the transmitter and relay are used to create a jamming signal in order to attack a passive eavesdropper. Also, they have assumed that PCSI is known at the relay and destination. The authors in [7], [8] have assumed that the source node in a cooperative system can send jamming signals to confuse the eavesdropper in a two-way relaying system without using an external jammer to increase the secrecy in the network. The cooperative jamming scheme of the three-phase full duplex relaying wiretap system has been represented in [9] to increase the secrecy capacity excluding an external jammer node and using one of the source nodes to interfere with the relay node signals. Many of researches have focused on secure MIMO communication systems when an eavesdropper can wiretap source-relay and relay-destination channels for partial and full cooperative jamming for two-hop DF relaying. Furthermore, inactive relay nodes are used as a jammer to send signals to interrupt the eavesdropper when PCSI is considered at an eavesdropper node [10].

In this paper, we propose a DF cooperative wireless system when a direct link is available between source and destination nodes. The channels in the network are slow and flat Rayleigh fading, independent and identically distributed. Also, an imperfect channel estimation at all nodes is considered. In addition, an active jammer node with a single antenna in the system is considered to create interference at the relay and destination nodes in all transmission phases when PCSI is unavailable at the relay and destination nodes. Two cooperative phases of transmission are considered in time division duplex. Finally, BER and throughput of the suggested system are derived for the imperfect channel state information (ICSI) and in the presence of jamming in the network.

The rest of this paper is organized as follows. The system model is described in Section II. In Section III, we analyze the BER of DF relaying in the presence of jamming. Throughput of the suggested system is given in section IV. In section V, numerical and simulation results are presented. Finally, we conclude this paper in Section VI.

\section{SYSTEM MODEL}

The system consists of four nodes, namely a source (S), a destination $(\mathrm{D})$, a relay $(\mathrm{R})$ and a jammer $(\mathrm{J})$. The source, relay 
and destination are all half-duplex DF single-antenna nodes. A direct link is available between the source and destination as shown in Fig.1. Furthermore, a jammer node with a single antenna is trying to attack the relay and destination nodes to degrade the performance of the system by adding interference to the received signals at the destination and relay. The parameters $h_{S, R}, h_{S, D}$ and $h_{R, D}$ are the complex channel coefficients between source-relay, source-destination and relay-destination respectively and model the slow flat Rayleigh fading. The channels are independent and identically distributed (i.i.d) and have similar fading average power gain with zero mean and variance $\sigma_{i, j}^{2}, i \in\{S, R\}$ and $j \in\{R, D\}$. The system transmission is considered to be orthogonal, either through time or frequency division (FD). For convenience, we assume time division (TD) where each node is assigned one time slot for each information packet. In the first time slot, the source node transmits signals to the relay and destination nodes. The relay node decodes and forwards the transmitted data to the destination node in the second time slot. In this work, the channels are estimated with error at relay and destination nodes. In addition, the bit duration is equal one , therefore the transmitted signal energy is equivalent to the transmitted power $\left(E_{i, j}=P_{i, j}\right), i \in\{S, R\}$ and $j \in\{R, D\}$. The received signals at the relay $y_{S, R}$ and at the destination $y_{S, D}$ nodes are given by

$$
\begin{gathered}
y_{S, R}=\sqrt{E_{S}} h_{S, R} x_{S}+\sqrt{E_{J}} h_{J, R} x_{J}+n_{S, R}, \\
y_{S, D}=\sqrt{E_{S}} h_{S, D} x_{S}+\sqrt{E_{J}} h_{J, D} x_{J}+n_{S, D} .
\end{gathered}
$$

The received signal from relay at the destination $y_{R, D}$ node is given by

$$
y_{R, D}=\sqrt{E_{R}} h_{R, D} x_{R}+\sqrt{E_{J}} h_{J, D} x_{J}+n_{R, D},
$$

where $x_{S}, x_{R}$, and $x_{J}$ denote the transmitted QPSK symbols at the source, relay and jammer respectively. $E_{S}, E_{R}$ and $E_{J}$ are the transmitted signal energies at the source, relay and jammer respectively. $n_{S, R}, n_{S, D}$ and $n_{R, D}$ are additive white Gaussian noises (AWGN) in the corresponding channels, with variance $\sigma_{S, R}^{2}, \sigma_{S, D}^{2}$ and $\sigma_{R, D}^{2}$ respectively. The channel $h_{i, j}$ is estimated at the receiver side to detect the incoming bits. Therefore the relationship between the channel and the estimated channel can be written as

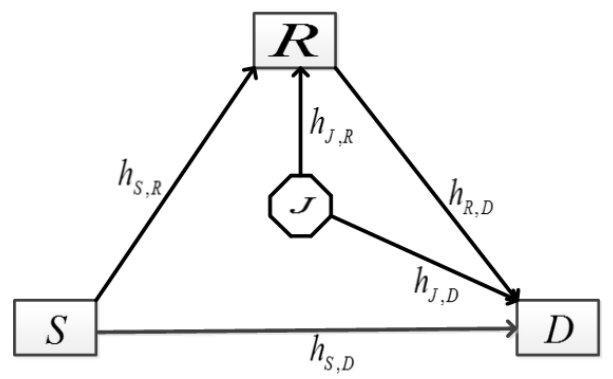

Fig. 1: Model of relaying system with a jammer node.

$$
\hat{h}_{i, j}=\left\{\begin{array}{l}
\rho_{i, j} h_{i, j}+\left(1-\rho_{i, j}\right) \zeta_{i, j} \text { if } \rho_{i, j} \neq 1 \\
h_{i, j} \text { if } \rho_{i, j}=1
\end{array}\right.
$$

where $\hat{h}_{i, j}$ is the estimated channel, $\rho_{i, j}$ is the correlation coefficient $\left(0 \leq \rho_{i, j} \leq 1\right), i \in\{S, R\}, j \in\{R, D\}$ and $\zeta_{i, j}$ is the AWGN in the corresponding estimated channels with variance $\sigma_{\zeta_{i, j}}^{2}$. The received signals at the relay and destination are compensated by the estimated channels given in (4), Therefore, the decision variable $\left(D_{i, j}\right)$ would be given by

$$
D_{i, j}=\Re\left\{y_{i, j} \hat{h}_{i, j}^{*}\right\},
$$

where $\Re$ is the real part, $\hat{h}_{i, j}^{*}$ is the complex conjugate of the estimated channel and $y_{i, j}, i, j \in\{S, R, D\}$ is the received signal at the node $j$. We can rewrite (1), (2) and (3) in general formula as

$$
y_{i, j}=\sqrt{E_{i}} h_{i, j} x_{i}+\sqrt{E_{J}} h_{J, j} x_{J}+n_{i, j} .
$$

Therefore, substituting (4) and (6) into (5), we can get

$$
\begin{aligned}
& D_{i, j}= \\
& \Re\left\{\left(\sqrt{E_{i}} h_{i, j} x_{i}+\sqrt{E_{J}} h_{J, j} x_{J}+n_{i, j}\right)\left(\rho_{i, j} h_{i, j}^{*}+\left(1-\rho_{i, j}\right) \zeta_{i, j}^{*}\right)\right\},
\end{aligned}
$$

where $h_{i, j}^{*}$ is the complex conjugate of the channel coefficient and $\zeta_{i, j}^{*}$ is the complex conjugate of the noise contained in the estimated channel.

\section{BER PERFORMANCE ANALYSIS}

In this section, the error probability of DF relaying in presence of jamming and imperfect channel estimation is derived in an exact form expression. The average error probability at the destination using maximal ratio combining (MRC) technique is given by [11]

$$
P_{e r r}(e)=P\left(\bar{e}_{S, R}\right) P\left(e_{S, D} \mid \bar{e}_{R, D}\right)+P\left(e_{S, R}\right) P\left(e_{S, D} \mid e_{R, D}\right),
$$

where, $P_{\text {err }}(e)$ is the end-to-end probability of error of DF at the destination node, $P\left(\bar{e}_{S, R}\right)$ is the probability that no error occurs during source-relay transmission, $P\left(e_{S, D} \mid \bar{e}_{R, D}\right)$ is the probability that an error occurs during sourcedestination transmission given that no error occurs during relay-destination transmission, $P\left(e_{S, R}\right)$ is the probability that an error occurs during source-relay transmission and $P\left(e_{S, D} \mid e_{R, D}\right)$ is the probability that an error occurs during source-destination transmission given that an error occurs during relay-destination transmission. Now, we find each component in eq. (8) as follows: For Rayleigh fading channel, the average bit error probability can be written as eq. (6.58) [12]

$$
P\left(e_{S, R}\right)=\frac{1}{2}\left(1-\sqrt{\frac{\bar{\gamma}_{S, R}}{1+\bar{\gamma}_{S, R}}}\right),
$$

where $\bar{\gamma}_{S, R}$ is the average SNR of the source-relay channel. To obtain the average SNR expression, we need to find the 
ratio of the signal power to the overall noise plus interference power, $\bar{\gamma}_{S, R}$, which can be written as

$$
\bar{\gamma}_{S, R}=\alpha_{S, R} / \beta_{S, R},
$$

where $\alpha_{S, R}$ is the signal power of the source-relay channel and $\beta_{S, R}$ is the noise plus interference of the source-relay channel. Based on eq. (7), the signal power at the relay node is given by

$$
\alpha_{S, R}=E_{S} \rho_{S, R}^{2} E\left(\left|h_{S, R}\right|^{2}\right)^{2} .
$$

and noise plus interference power at the relay node from source and jammer can be written as

$$
\begin{aligned}
& \beta_{S, R}=\underbrace{E_{S}\left(1-\rho_{S, R}\right)^{2} E\left(\left|h_{S, R}\right|^{2}\right) \sigma_{\zeta_{S, R}}^{2}}_{A 1} \\
& +\underbrace{\rho_{S, R}^{2} E\left(\left|h_{S, R}\right|^{2}\right) \sigma_{n_{S, R}^{2}}^{2}+\left(1-\rho_{S, R}\right)^{2} \sigma_{\zeta_{S, R}}^{2} \sigma_{n_{S, R}}^{2}}_{C 1} \\
& +\underbrace{E_{J} \rho_{S, R}^{2} E\left(\left|h_{S, R}\right|^{2}\right) E\left(\left|h_{J, R}\right|^{2}\right)}_{D 1} \\
& +\underbrace{E_{J}\left(1-\rho_{S, R}\right)^{2} E\left(\left|h_{J, R}\right|^{2}\right) \sigma_{\zeta_{S, R}}^{2}}_{C 1} .
\end{aligned}
$$

where $E($.$) is the expectation operator. From eq. (12), it should$ be noticed that there are three kinds of interferences in the network. First interference (A1) represents the impact of the estimated channel on the useful signal at the relay, the second interference (B1) occurs as a result of multiplication of noise in eq. (1) with eq. (4) at the relay and the third interference (C1) and (D1) represents the impact of the jamming signal with estimated channel on the relay. Substituting (12) (11), (12) into (10) we can have an expression for the SNR $\bar{\gamma}_{S R}$ for source-relay channel. Therefore, the probability that no error occurs during source-relay transmission $P\left(\bar{e}_{S, R}\right)$ would be given by

$$
\begin{aligned}
P\left(\bar{e}_{S, R}\right) & =1-P\left(e_{S, R}\right) \\
& =\frac{1}{2}\left(1+\sqrt{\frac{\bar{\gamma}_{S, R}}{1+\bar{\gamma}_{S, R}}}\right),
\end{aligned}
$$

In case of a correct decoding at $\mathrm{R}$, the symbols are equal $x_{R}=x_{S}$. Therefore, the receiver becomes equivalent to MRC with two paths. In this case, $P\left(e_{S, D} \mid \bar{e}_{R, D}\right)$ can be written as eq. (7.20) [12] for $M=2$ ( $M$ is number of branches at the destination)

$$
\begin{array}{r}
P\left(e_{S, D} \mid \bar{e}_{R, D}\right)=\frac{1}{2^{2}}\left(1-\sqrt{\frac{\bar{\gamma}_{S, R, D}}{1+\bar{\gamma}_{S, R, D}}}\right)^{2} \times \\
{\left[1+\frac{1}{2}\left(1+\sqrt{\frac{\bar{\gamma}_{S, R, D}}{1+\bar{\gamma}_{S, R, D}}}\right)\right],}
\end{array}
$$

and $\bar{\gamma}_{S, R, D}$ is given by

$$
\bar{\gamma}_{S, R, D}=\frac{\alpha_{S, D}+\alpha_{R, D}}{\beta_{S, D}+\beta_{R, D}}
$$

where $\bar{\gamma}_{S, R, D}$ is the average SNR for the equivalent MRC scheme obtained for direct and indirect links, $\alpha_{S, D}$ is the signal power of source-destination channel, $\alpha_{R, D}$ is the signal power of relay-destination channel, $\beta_{S, D}$ is the noise plus interference of source-destination channel and $\beta_{R, D}$ is the noise plus interference of relay-destination channel.

Therefore, signal power for $S \rightarrow D$ link can be written as

$$
\alpha_{S, D}=E_{S} \rho_{S, D}^{2} E\left(\left|h_{S, D}\right|^{2}\right)^{2} .
$$

and signal power for $R \rightarrow D$ link is given by

$$
\alpha_{R, D}=E_{R} \rho_{R, D}^{2} E\left(\left|h_{R, D}\right|^{2}\right)^{2} .
$$

Similarly, for the $S \rightarrow D$ and $R \rightarrow D$ links, we have

$$
\begin{aligned}
& \beta_{S, D}=\underbrace{E_{S}\left(1-\rho_{S, D}\right)^{2} E\left(\left|h_{S, D}\right|^{2}\right) \sigma_{\zeta_{S, D}}^{2}}_{A 2} \\
& +\underbrace{\rho_{S, D}^{2} E\left(\left|h_{S, D}\right|^{2}\right) \sigma_{n_{S, D}}^{2}+E\left(1-\rho_{S, D}\right)^{2} \sigma_{\zeta_{S, D}}^{2} \sigma_{n_{S, D}}^{2}}_{B 2} \\
& +\underbrace{E_{J} \rho_{S, D}^{2} E\left(\left|h_{S, D}\right|^{2}\right) E\left(\left|h_{J, D}\right|^{2}\right)}_{C 2} \\
& +\underbrace{E_{J}\left(1-\rho_{S, D}\right)^{2} E\left(\left|h_{J, D}\right|^{2}\right) \sigma_{\zeta_{S, D}}^{2}}_{D 2} . \\
& \beta_{R, D}=\underbrace{E_{R}\left(1-\rho_{R, D}\right)^{2} E\left(\left|h_{R, D}\right|^{2}\right) \sigma_{\zeta_{R, D}}^{2}}_{A 3} \\
& +\underbrace{\rho_{R, D}^{2} E\left(\left|h_{R, D}\right|^{2}\right) \sigma_{n_{R, D}}^{2}+\left(1-\rho_{R, D}\right)^{2} \sigma_{\zeta_{R, D}}^{2} \sigma_{n_{R, D}}^{2}}_{B 3} \\
& +\underbrace{E_{J} \rho_{R, D}^{2} E\left(\left|h_{R, D}\right|^{2}\right) E\left(\left|h_{J, D}\right|^{2}\right)}_{C 3} \\
& +\underbrace{E_{J}\left(1-\rho_{R, D}\right)^{2} E\left(\left|h_{J, D}\right|^{2}\right) \sigma_{\zeta_{R, D}}^{2}}_{D 3} .
\end{aligned}
$$

All terms in (18) and (19) are similar to the terms in (12) except that they take place in the $S \rightarrow D$ and $R \rightarrow D$ links. Substituting (16), (17), (18) and (19) into (15) and then substituting into (14), we can have an expression of $P\left(e_{S, D} \mid \bar{e}_{R, D}\right)$.

In case of an erroneous decision at $\mathrm{R}$, the symbols are complemented such that $x_{R}=-x_{S}$ which causes the received signals at the relay to be decoded incorrectly and forwarded to the destination node such that (3) becomes

$$
y_{R, D}=-\sqrt{E_{R}} h_{R, D} x_{s}+\sqrt{E_{J}} h_{J, D} x_{J}+n_{R, D} .
$$

The assumption that the channels have equal average power gain $\left(E\left(\left|h_{S, D}\right|^{2}\right)=E\left(\left|h_{R, D}\right|^{2}\right)\right)$ gives an average SNR, $\bar{\gamma}_{S, R, D}=0$. Hence, the $P\left(e_{S, D} \mid e_{R, D}\right)$ can be written as

$$
P\left(e_{S, D} \mid e_{R, D}\right)=0.5
$$

Substituting (9), (13), (14) and (21) into (8), we can have an expression for $P_{e r r}(e)$ which is written as in (22) on the next page.

\section{THROUGHPUT}

In this section, we present the performance of the throughput of cooperative DF relaying. The throughput of the system is defined as the total number of packets that are successfully delivered over the channel in the network for a given period of time [13], [14].

$$
G=R_{T} / E(T)
$$


$P_{e r r}(e)=\frac{1}{2}\left(1+\sqrt{\frac{\bar{\gamma}_{S, R}}{1+\bar{\gamma}_{S, R}}}\right) \times \frac{1}{2^{2}}\left(1-\sqrt{\frac{\bar{\gamma}_{S, R, D}}{1+\bar{\gamma}_{S, R, D}}}\right)^{2}\left[1+\frac{1}{2}\left(1+\sqrt{\frac{\bar{\gamma}_{S, R, D}}{1+\bar{\gamma}_{S, R, D}}}\right)\right]+\frac{1}{2}\left(1-\sqrt{\frac{\bar{\gamma}_{S, R}}{1+\bar{\gamma}_{S, R}}}\right) \times 0.5$

where $G$ is the throughput (bits/sec), $R_{T}$ is the target rate (bits/sec/Hz) and $E(T)$ is the average delay. The expected number of transmissions (original transmission plus retransmissions) is given by [13]

$$
E(T)=\sum_{n=1}^{\infty} n P\{T=n\}
$$

where $n$ is the number of successful transmissions and $P\{T=n\}$ is the probability of successful transmission after $T$ time slots. In our work, we have one time slot for direct transmission and two time slots for indirect transmission through the relay. In the first phase of the transmission which occurs at $T=1$ over $S \rightarrow D$ channel, the probability of successful transmission becomes

$P_{r}(T=1)=P_{r}\left(\bar{\gamma}_{S, D}>\gamma_{o}\right)=1-P_{r}\left(\bar{\gamma}_{S, D} \leq \gamma_{o}\right)=e^{\left(\frac{-\gamma_{o}}{\bar{\gamma}_{S, D}}\right)}$,

where $\gamma_{o}$ is the decision threshold. In the second phase of the transmission $T=2$ over $R \rightarrow D$ channel, the probability of successful transmission becomes

$$
P_{r}(T=2)=P_{r}\left(\bar{\gamma}_{S, D}<\gamma_{o}\right)=1-e^{\left(\frac{-\gamma_{o}}{\bar{\gamma}_{S, D}}\right)} .
$$

Substituting (25) and (26) into (24), and then substituting into (23), the throughput of the proposed system can be written as

$$
G=\frac{R_{T}}{2-e^{\left(\frac{-\gamma_{o}}{\bar{\gamma}_{S, D}}\right)}} .
$$

\section{Simulation Results}

The simulation results are presented to confirm the exactitude of our derivation of BER, throughput and evaluate the impact of jamming with ICSI. The channels $h_{S, D}, h_{S, R}$ and $h_{R, D}$ are assumed to be zero-mean complex Gaussian random variables with equal variance $\sigma_{i, j}^{2}=1, \sigma_{i, j}^{2}, i \in\{S, R\}$, $j \in\{R, D\}$ and the transmitted signal energy at the source $E_{S}=1$. QPSK modulation is used in which the BER and throughput of the system versus SNR are considered as the performance metrics. Fig. 2 compares the probability of error versus the SNR of the proposed system in case of PCSI and ICSI with different values of $\rho_{i, j}$ without a jammer. A perfect matching between theoretical and simulation results is shown for all scenarios. It should also be noticed that the degradation resulting in terms of BER using ICSI is due to the decrease of $\rho_{i, j}$ values. It is noticed that the BER is very sensitive to $\rho_{i, j}$ especially at high SNR. Fig. 3 shows the impact of jamming and ICSI on the BER performance. Adding a jammer node to the network will degrade BER performance in both cases of PCSI and ICSI because the noise will be increased. In addition, we can see that decreasing $\rho_{i, j}$ leads to increasing the interference at the destination which causes BER performance degradation as deduced from eq. (7). Fig. 4 illustrates the BER performance of the proposed cooperative wireless scheme for several values of $E_{J_{i, j}}$, where $E_{J_{i, j}}$ is the transmitted energy at the jammer on $i \rightarrow j$ channel. Also, different locations of the jammer in the network are simulated. In the first case, we assume the jammer is close to destination and the energy of the jammer is high for $S \rightarrow D$ and $R \rightarrow D$ branches and is low for $S \rightarrow R,\left(E_{J_{S, D}}, E_{J_{S, R}}, E_{J_{R, D}}\right)=(0.9,0.4,0.9)$ , which decreases the BER. In the second case, we assume the jammer is close to the source or the relay and $\left(E_{J_{S, D}}, E_{J_{S, R}}, E_{J_{R, D}}\right)=(0.9,0.9,0.4),(0.4,0.9,0.9)$. In this case, BER performance is very close as shown in Fig. 4, this is expected as narrated by eq. (13). In the third case, the jammer node is in the middle of the network and has equal distances to other nodes $\left(E_{J_{S, D}}, E_{J_{S, R}}, E_{J_{R, D}}\right)=(0.9,0.9,0.9)$ which constitutes the worst case scenario for BER performance. Fig. 5 plots the throughput of the proposed system versus SNR for different threshold values $\gamma_{o}$ in these cases, PCSI, ICSI and ICSI with a jammer. From Fig. 5, we can conclude that PCSI gives high throughput while in case of ICSI with and without a jammer gives low throughput at low and medium SNR. This is a significant advantage if an eavesdropper attacks the relaying system.

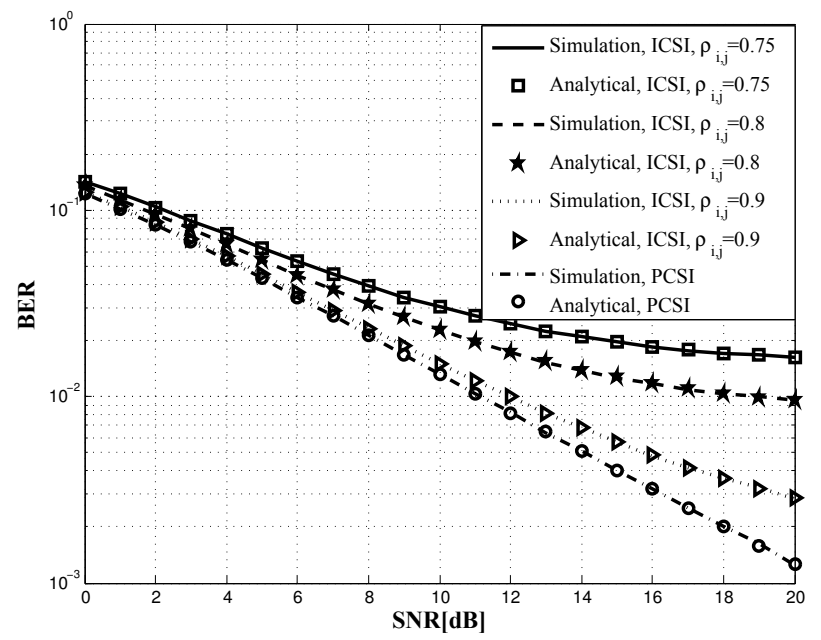

Fig. 2: Simulation and analytical BER of cooperative scheme for different values of $\rho_{i, j}$

\section{CONCLUSION}

In this paper, a single DF cooperative relaying scheme in the presence of jamming with imperfect channel state information is studied and analysed. Taking into account the estimation error and jamming in the network, BER expression is derived for independent and identical slow and flat Rayleigh fading 


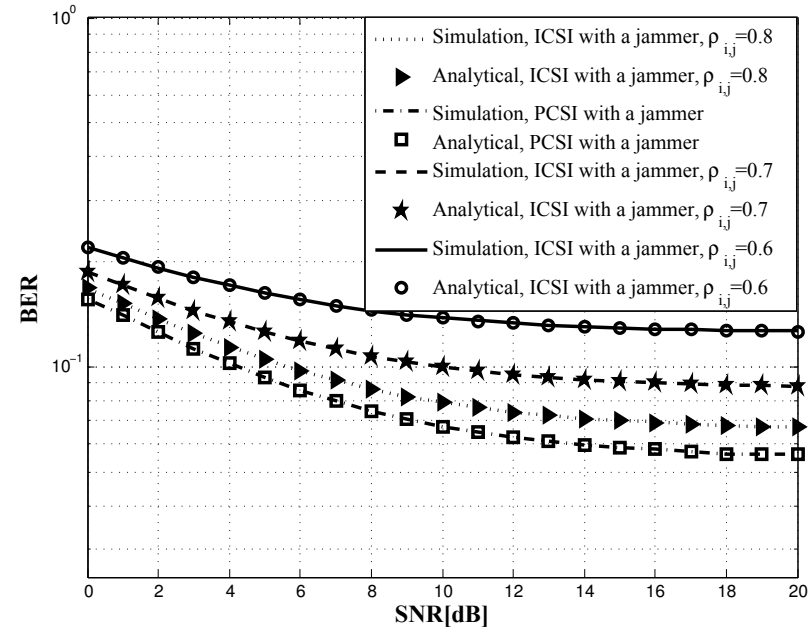

Fig. 3: Simulation and analytical BER of cooperative scheme for different values of $\rho_{i, j}$ and $E_{j}=0.4$

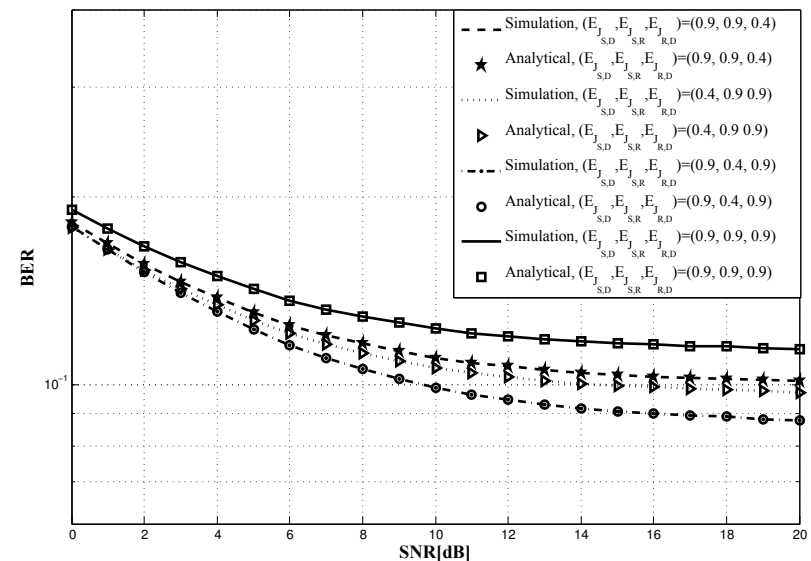

Fig. 4: Simulation and analytical BER of cooperative scheme for different values of $\left(E_{J_{S, D}}, E_{J_{S, R}}, E_{J_{R, D}}\right)$ and $\rho_{i, j}=0.95$

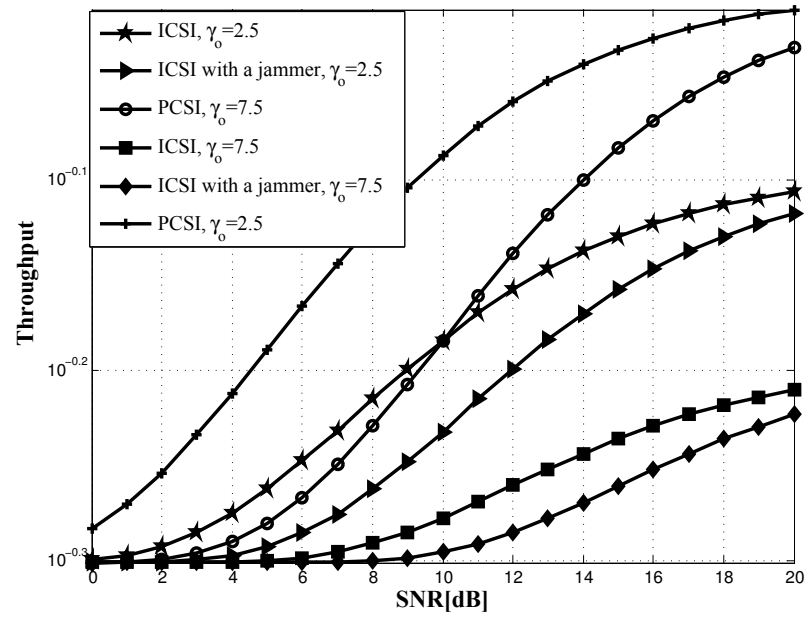

Fig. 5: Throughput of cooperative scheme for different values of $\gamma_{o}$ and $\rho_{S, D}=0.75$ channels with imperfect channel estimation. Furthermore, the throughput of the proposed system is obtained in this paper. Results show that BER performance with ICSI and a jammer node is degraded and gives high probability of error because the channel estimated and the jammer interferences in the network. The worst case scenario for BER performance occurs when the jammer is located in the middle of the network and has equal distances to all nodes. Our results represent the throughput of the suggested scheme and show that the throughput is low at low and medium SNR and is dependent on the decision threshold.

\section{ACKNOWLEDGMENT}

This work has been supported by the NSERC discovery grant $435243-2013$.

\section{REFERENCES}

[1] J. N. Laneman, D. N. Tse, and G. W. Wornell, "Cooperative diversity in wireless networks: Efficient protocols and outage behavior," IEEE Trans. on Info. Theory, vol. 50, no. 12, pp. 3062-3080, 2004.

[2] A. Sendonaris, E. Erkip, and B. Aazhang, "User cooperation diversity. part I. system description," IEEE Trans. on Commun., vol. 51, no. 11, pp. 1927-1938, 2003

[3] — "User cooperation diversity. part II. implementation aspects and performance analysis," IEEE Trans. on Commun., vol. 51, no. 11, pp. 1939-1948, 2003.

[4] X. Zhou, M. Qiu, S.-C. Lin, and Y.-W. P. Hong, "On the jamming power allocation and signal design in df relay networks," in IEEE Signals, Systems and Computers, 2013 Asilomar Conference on, 2013, pp. 12681272.

[5] E. Altman, K. Avrachenkov, and A. Garnaev, "Jamming in wireless networks under uncertainty," Mobile Networks and Applications, vol. 16, no. 2 , pp. 246-254, 2011.

[6] L. Dong, H. Yousefi'zadeh, and H. Jafarkhani, "Cooperative jamming and power allocation for wireless relay networks in presence of eavesdropper," in IEEE International Conference on Communications (ICC), 2011, pp. 1-5

[7] H. Long, W. Xiang, J. Wang, Y. Zhang, H. Zhao, and W. Wang, "Cooperative jamming and power allocation in two-way relaying system with eavesdropper," in IEEE Vehicular Technology Conference (VTC Fall), 2013, pp. 1-5.

[8] H. Long, W. Xiang, J. Wang, Y. Zhang, and W. Wang, "Cooperative jamming and power allocation with untrusty two-way relay nodes," IET Commun., vol. 8, no. 13, pp. 2290-2297, 2014.

[9] H. Long, W. Xiange, X. Zhang, J. Wang, and W. Wang, "Cooperative jamming and power allocation in three-phase two-way relaying system with untrusty relay node," in IEEE General Assembly and Scientific Symposium (URSI GASS), 2014 XXXIth URSI, 2014, pp. 1-4.

[10] J. Huang and A. L. Swindlehurst, "Cooperative jamming for secure communications in mimo relay networks," IEEE Signal Processing, Trans. on, vol. 59, no. 10, pp. 4871-4884, 2011.

[11] M. Wu, D. Wubben, and A. Dekorsy, "BER-based power allocation for decode-and-forward relaying with m-qam constellations," in IEEE Wireless Communications and Mobile Computing Conference (IWCMC), 2011, pp. 888-893.

[12] A. Goldsmith, Wireless communications. Cambridge University Press, 2005.

[13] I. Stanojev, O. Simeone, Y. Bar-Ness, and C. You, "Performance of multi-relay collaborative hybrid-arq protocols over fading channels," IEEE Commun. Letters, vol. 10, no. 7, pp. 522-524, 2006.

[14] S. S. Ikki and M. H. Ahmed, "Performance analysis of decodeand-forward incremental relaying cooperative-diversity networks over rayleigh fading channels," in IEEE Vehicular Technology Conference

(VTC Spring), 2009, pp. 1-6. 